\title{
Three-point functions in duality-invariant higher-derivative gravity
}

\author{
Usman Naseer and Barton Zwiebach \\ Center for Theoretical Physics, Massachusetts Institute of Technology, \\ 77 Massachusetts Avenue, Cambridge, MA 02139, U.S.A. \\ E-mail: unaseer@mit.edu, zwiebach@mit.edu
}

ABSTRACT: Doubled $\alpha^{\prime}$-geometry is the simplest higher-derivative gravitational theory with exact global duality symmetry. We use the double metric formulation of this theory to compute on-shell three-point functions to all orders in $\alpha^{\prime}$. A simple pattern emerges when comparing with the analogous bosonic and heterotic three-point functions. As in these theories, the amplitudes factorize. The theory has no Gauss-Bonnet term, but contains a Riemann-cubed interaction to second order in $\alpha^{\prime}$.

Keywords: Global Symmetries, Models of Quantum Gravity, Scattering Amplitudes, String Duality

ArXiv ePrint: 1602.01101 


\section{Contents}

1 Introduction $\quad 1$

2 Bosonic, heterotic, and HSZ three-point amplitudes 2

3 Derivative expansion of HSZ theory 5

4 Perturbative expansion of HSZ theory $\quad 9$

4.1 Perturbative expansion of the two-derivative Lagrangian 9

$\begin{array}{lll}4.2 & \text { General treatment of auxiliary fields } & 12\end{array}$

$\begin{array}{lll}4.3 & \text { Higher-derivative Lagrangian and on-shell amplitudes } & 13\end{array}$

5 Conclusions and remarks $\quad 16$

\section{Introduction}

It is well known that the discrete duality symmetries of toroidally compactified string theories imply continuous duality symmetries of the classical effective field theory for the massless string degrees of freedom [1-4]. Double field theory formulates the higher-dimensional two-derivative massless effective field theory in a way that the duality symmetry can be anticipated before dimensional reduction [5-9]. When higher-derivative corrections (or $\alpha^{\prime}$ corrections) are included it becomes much harder to provide a duality covariant formulation. It is generally expected that as soon as higher-derivatives are included, all numbers of them are required for exact duality invariance.

At present, there is only one known example of an effective gravitational theory with higher-derivatives and exact duality invariance: the "doubled $\alpha^{\prime}$ geometry" of Hohm, Siegel and Zwiebach, henceforth called HSZ theory [10]. Two key facts about this theory are relevant to our discussion. First, its spacetime Lagrangian is efficiently written in terms of a double metric $\mathcal{M}$, an unconstrained version of the generalized metric $\mathcal{H}$ which encodes the metric $g$ and the antisymmetric field $b$ in a familiar fashion. The Lagrangian is cubic in $\mathcal{M}$ and includes terms with up to six derivatives. In $\mathcal{H}$ variables, however, the Lagrangian has terms of all orders in derivatives [11]. Second, HSZ theory is not the low-energy effective field theory of bosonic strings, nor that of heterotic strings. It does not contain gauge fields, but due to the Green-Schwarz modification of the gauge transformations of the $b$ field, it contains higher-derivative terms such as a Chern-Simons modification of the field strength $H$ for $b$ [12]. A gauge principle to accommodate higher-derivative corrections of bosonic and heterotic strings has been investigated in [13].

The purpose of this paper is to calculate the simplest amplitudes in HSZ theory; onshell three-point amplitudes for the metric and $b$ field. While this is a relatively simple 
matter in any gravitational theory described in terms of a metric and a $b$ field, it is a rather nontrivial computation in a theory formulated in terms of a double metric $\mathcal{M} .^{1}$ This is so because metric and $b$ field fluctuations are encoded nontrivially in $\mathcal{M}$ fluctuations and because $\mathcal{M}$ also contains unfamiliar auxiliary fields. These amplitudes, not yet known, will be obtained using the $\mathcal{M}$ field Lagrangian. The procedure is instructive: it requires us to obtain the explicit $\alpha^{\prime}$ expansion of the Lagrangian and to discuss the elimination of auxiliary fields. The three-point amplitudes turn out to be simple, suggesting that higher point functions should be calculable. We suspect that world-sheet methods will eventually prove superior for the computation of general amplitudes. In fact, reference [10] discussed how the chiral world-sheet theory appears to be a singular limit of the conventional world sheet theory, and the recent elaboration in [14] goes further in this direction and discusses amplitudes. Our results provide a test of world-sheet methods for the simplest case. There are other works on amplitudes motivated by or making use of double field theory $[15,16]$.

In both bosonic string theory and heterotic theory, on-shell three-point amplitudes factorize into factors that involve left-handed indices and right-handed indices (see eq. (2.4)). We show that in HSZ these amplitudes also factorize (see eq. (2.5)). The explicit form of the result has implications for the low-energy effective field theory. In the bosonic string the terms in the low-energy effective action needed to reproduce its three-point amplitudes include Riemann-squared (or Gauss-Bonnet) $[17,18]$ and $H H R$ terms to first order in $\alpha^{\prime}$, and Riemann-cubed to second order in $\alpha^{\prime}[19,20]$. To reproduce the (gravitational) heterotic three-point amplitudes the theory has only order $\alpha^{\prime}$ terms: Gauss-Bonnet, HHR and a $b$-odd term $b \Gamma \partial \Gamma$, with $\Gamma$ the Christoffel connection. At order $\alpha^{\prime}$ HSZ theory contains only the $b$-odd term with twice the coefficient in heterotic string, and to second order in $\alpha^{\prime}$ the bosonic string Riemann-cubed term with opposite sign. Our work shows that to order $\left(\alpha^{\prime}\right)^{2}$, the following is the gauge invariant action that reproduces the on-shell cubic amplitudes of HSZ theory:

$$
S=\int d^{D} x \sqrt{-g} e^{-2 \phi}\left(R+4(\partial \phi)^{2}-\frac{1}{12} \widehat{H}_{i j k} \widehat{H}^{i j k}-\frac{1}{48} \alpha^{\prime 2} R_{\mu \nu}{ }^{\alpha \beta} R_{\alpha \beta}{ }^{\rho \sigma} R_{\rho \sigma}{ }^{\mu \nu}\right) .
$$

The $\mathcal{O}\left(\alpha^{\prime}\right)$ terms above arise from the kinetic term for the three-form curvature [21]. We have $\widehat{H}_{i j k}=H_{i j k}+3 \alpha^{\prime} \Omega_{i j k}(\Gamma)$, where $H_{i j k}=3 \partial_{[i} b_{j k]}$ with the Chern Simons term $\Omega$ given by:

$$
\Omega_{i j k}(\Gamma)=\Gamma_{[i|p|}^{q} \partial_{j} \Gamma_{k] q}^{p}+\frac{2}{3} \Gamma_{[i|p|}^{q} \Gamma_{[j|r|}^{p} \Gamma_{[k] q \mid}^{r} .
$$

In the conclusion section we discuss possible calculations that may advance our understanding of duality-invariant higher-derivative field theories.

\section{Bosonic, heterotic, and HSZ three-point amplitudes}

In this section we motivate and state our main claim: in HSZ theory, on-shell three-point amplitude for gravity and $b$ fields exhibits a factorization structure analogous to that of the

\footnotetext{
${ }^{1}$ The computation in terms of $\mathcal{H}$ variables would not be practical, as even the terms with four derivatives have not been explicitly written out.
} 
bosonic and heterotic string. For this purpose let us consider these amplitudes. Let $k_{1}, k_{2}$, and $k_{3}$ denote the momenta of the particles. Since we are dealing with massless states, the on-shell condition and momentum conservation imply that for all values of $a, b=1,2,3$ :

$$
k_{a} \cdot k_{b}=0 .
$$

We also have three polarization tensors $e_{a i j}$ with $a=1,2,3$. Symmetric traceless polarizations represent gravitons, and antisymmetric polarizations represent $b$ fields. Dilaton states are encoded by polarizations proportional to the Minkowski metric [22]. The polarizations satisfy transversality

$$
k_{a}^{i} e_{a i j}=0, \quad k_{a}^{j} e_{a i j}=0, \quad a \text { not summed. }
$$

To construct the three-point amplitudes one defines the auxiliary three-index tensors $T$ and $W$ :

$$
\begin{aligned}
T^{i j k}\left(k_{1}, k_{2}, k_{3}\right) & \equiv \eta^{i j} k_{12}^{k}+\eta^{j k} k_{23}^{i}+\eta^{k i} k_{31}^{j}, \\
W^{i j k}\left(k_{1}, k_{2}, k_{3}\right) & \equiv \frac{1}{8} \alpha^{\prime} k_{23}^{i} k_{31}^{j} k_{12}^{k},
\end{aligned}
$$

with $k_{a b}=k_{a}-k_{b}$. Note the invariance of $T$ and $W$ under simultaneous cyclic shifts of the spacetime indices and the 1,2,3 labels. For bosonic and heterotic strings the on-shell amplitudes for three massless closed string states with polarizations $e_{a i j}$ are given by (see, for example, eq. (6.6.19) in [23] and eq. (12.4.14) in [24]):

$$
\begin{aligned}
S_{\mathrm{bos}} & =\frac{i}{2} \kappa(2 \pi)^{D} \delta^{D}\left(\sum p\right) e_{1 i i^{\prime}} e_{2 j j^{\prime}} e_{3 k k^{\prime}}(T+W)^{i j k}(T+W)^{i^{\prime} j^{\prime} k^{\prime}}, \\
S_{\mathrm{het}} & =\frac{i}{2} \kappa(2 \pi)^{D} \delta^{D}\left(\sum p\right) e_{1 i i^{\prime}} e_{2 j j^{\prime}} e_{3 k k^{\prime}}(T+W)^{i j k} T^{i^{\prime} j^{\prime} k^{\prime}} .
\end{aligned}
$$

Note the factorization of the amplitude into a factor that involves the first indices on the polarization tensors and a factor that involves the second indices on the polarization tensors. $^{2}$ We claim that in HSZ theory the on-shell amplitudes also factorize:

$$
S_{\mathrm{hsz}}=\frac{i}{2} \kappa(2 \pi)^{D} \delta^{D}\left(\sum p\right) e_{1 i i^{\prime}} e_{2 j j^{\prime}} e_{3 k k^{\prime}}(T+W)^{i j k}(T-W)^{i^{\prime} j^{\prime} k^{\prime}} .
$$

For the bosonic string $(T+W)^{i j k}(T+W)^{i^{\prime} j^{\prime} k^{\prime}}$ is symmetric under the simultaneous exchange of primed and unprimed indices. As a result, the amplitude for any odd number of $b$ fields vanishes. Expanding out

$$
\begin{aligned}
S_{\mathrm{bos}}= & \frac{i}{2} \kappa(2 \pi)^{D} \delta^{D}\left(\sum p\right) e_{1 i i^{\prime}} e_{2 j j^{\prime}} e_{3 k k^{\prime}} \\
& \times\left(T^{i j k} T^{i^{\prime} j^{\prime} k^{\prime}}+\left[W^{i j k} T^{i^{\prime} j^{\prime} k^{\prime}}+W^{i^{\prime} j^{\prime} k^{\prime}} T^{i j k}\right]+W^{i j k} W^{i^{\prime} j^{\prime} k^{\prime}}\right),
\end{aligned}
$$

\footnotetext{
${ }^{2}$ The on-shell conditions satisfied by the momenta imply that there are no candidates for three-point amplitudes with more than six derivatives.
} 
making clear the separation into two-, four-, and six-derivative structures, all of which are separately invariant under the simultaneous exchange of primed and unprimed indices. The four-derivative structure indicates the presence of Riemann-squared or GaussBonnet terms $[17,18]$. The six-derivative structure implies the presence of Riemann-cubed terms [20]. For the heterotic string we write the amplitude as

$$
\begin{aligned}
S_{\mathrm{het}}= & \frac{i}{2} \kappa(2 \pi)^{D} \delta^{D}\left(\sum p\right) e_{1 i i^{\prime}} e_{2 j j^{\prime}} e_{3 k k^{\prime}}\left(T^{i j k} T^{i^{\prime} j^{\prime} k^{\prime}}+\frac{1}{2}\left[W^{i j k} T^{i^{\prime} j^{\prime} k^{\prime}}+W^{i^{\prime} j^{\prime} k^{\prime}} T^{i j k}\right]\right. \\
& \left.+\frac{1}{2}\left[W^{i j k} T^{i^{\prime} j^{\prime} k^{\prime}}-W^{i^{\prime} j^{\prime} k^{\prime}} T^{i j k}\right]\right) .
\end{aligned}
$$

We have split the four-derivative terms into a first group, symmetric under the simultaneous exchange of primed and unprimed indices, and a second group, antisymmetric under the simultaneous exchange of primed and unprimed indices. The first group is one-half of the four-derivative terms in bosonic string theory, a well-known result. The second group represents four-derivative terms that can only have an odd number of $b$ fields. In fact, only one $b$ field is allowed. The term with three $b$ fields would have to be of the form $H Н \partial H$, with $H=d b$ and it can be shown to vanish by Bianchi identities. The term that one gets is of the form $Н Г \partial Г$, and arises from the kinetic term of the Chern-Simons corrected $b$-field field strength. This kind of term also appears in HSZ theory, as discussed in [11].

Expanding the HSZ amplitude above one finds

$$
\begin{aligned}
S_{\mathrm{hsz}}= & \frac{i}{2} \kappa(2 \pi)^{D} \delta^{D}\left(\sum p\right) e_{1 i i^{\prime}} e_{2 j j^{\prime}} e_{3 k k^{\prime}} \\
& \times\left(T^{i j k} T^{i^{\prime} j^{\prime} k^{\prime}}+\left[W^{i j k} T^{i^{\prime} j^{\prime} k^{\prime}}-T^{i j k} W^{i^{\prime} j^{\prime} k^{\prime}}\right]-W^{i j k} W^{i^{\prime} j^{\prime} k^{\prime}}\right),
\end{aligned}
$$

implying that there is no Gauss-Bonnet term, that the term with four derivatives has a single $b$ field and is the same as in heterotic string but with twice the magnitude. The six-derivative term is the same as in bosonic string, but with opposite sign. This implies that the Riemann-cubed term in the HSZ action and in bosonic strings have opposite signs. Most of the work in the rest of the paper deals with the computation of the $g$ and $b$ three-point amplitudes that confirms (2.8) holds.

It is useful to have simplified expressions for the amplitudes. For later use we record the following results, with 'cyc.' indicating that two copies of the terms to the left must be added with cyclic permutations of the 1,2 , and 3 labels:

$$
\begin{aligned}
e_{1 i i^{\prime}} e_{2 j j^{\prime}} e_{3 k k^{\prime}} T^{i j k} T^{i^{\prime} j^{\prime} k^{\prime}} & =\operatorname{tr}\left(e_{1}^{T} e_{2}\right)\left(k_{12} e_{3} k_{12}\right)+k_{12}\left(e_{3} e_{2}^{T} e_{1}+e_{3}^{T} e_{2} e_{1}^{T}\right) k_{23}+\text { cyc. } \\
e_{1 i i^{\prime}} e_{2 j j^{\prime}} e_{3 k k^{\prime}}\left(W^{i j k} T^{i^{\prime} j^{\prime} k^{\prime}} \pm T^{i j k} W^{i^{\prime} j^{\prime} k^{\prime}}\right) & =\frac{1}{8} \alpha^{\prime}\left[k_{12}\left(e_{3} e_{1}^{T} \pm e_{3}^{T} e_{1}\right) k_{23}\left(k_{31} e_{2} k_{31}\right)+\text { cyc. }\right], \\
e_{1 i i^{\prime}} e_{2 j j^{\prime}} e_{3 k k^{\prime}} W^{i j k} W^{i^{\prime} j^{\prime} k^{\prime}} & =\frac{1}{64} \alpha^{\prime 2}\left(k_{12} e_{3} k_{12}\right)\left(k_{23} e_{1} k_{23}\right)\left(k_{31} e_{2} k_{31}\right) .
\end{aligned}
$$

The formulae (2.4) for massless on-shell three-point amplitudes also hold for amplitudes that involve the dilaton. For the dilaton one must use a polarization tensor proportional to the Minkowski metric. Although we will not use the HSZ action to compute dilaton 
amplitudes, the predictions from the factorized amplitude (2.5) are exactly what we expect for the the dilaton. We explain this now.

Let $\hat{\phi}$ denote the physical dilaton field. For cubic dilaton interactions $\hat{\phi}^{3}$ there is no on-shell candidate at two, four, or six derivatives. For $\hat{\phi}^{2} e$ interactions there is no onshell candidate at four or six derivatives, but there is one at two derivatives: $\partial^{i} \hat{\phi} \partial^{j} \hat{\phi} e_{i j} \sim$ $\partial^{i} \hat{\phi} \partial^{j} \hat{\phi} h_{i j}$. This term does arise from the first line in (2.9) when we take $e_{1 i i^{\prime}} \sim \eta_{i i^{\prime}} \hat{\phi}$, $e_{2 j j^{\prime}} \sim \eta_{j j^{\prime}} \hat{\phi}$, and $e_{3 k k^{\prime}}=h_{k k^{\prime}}$. It is present in all three theories as it is the universal coupling of a scalar to gravity.

For $\hat{\phi} e e$ there are no on-shell candidates with six derivatives, but there are candidates with two and with four derivatives. Let's consider first the on-shell candidates with two derivatives. Again, an examination of the first line in (2.9) shows that $\hat{\phi} h h$ vanishes. This is expected: the physical dilaton does not couple to the scalar curvature. There is also no $\hat{\phi} h b$ coupling. On the other hand one can check that $\hat{\phi} b b$ does not vanish. This is also expected, as an exponential of $\hat{\phi}$ multiplies the $b$-field kinetic term. Again, all this is valid for the three theories.

Let us now consider $\hat{\phi} e e$ on-shell couplings with four derivatives. There is just one onshell candidate: $\hat{\phi} \partial^{i j} e_{k l} \partial^{k l} e_{i j}$. Due to the commutativity of derivatives this term requires both $e$ 's to be gravitons. This coupling arises both in bosonic and heterotic string theory because an exponential of $\hat{\phi}$ multiplies Riemann-squared terms. As expected, it can be seen from the second line in (2.9), using the top sign. It does not arise in HSZ theory because in this theory the four-derivative terms are odd under the $\mathbb{Z}_{2}$ transformation $b \rightarrow-b$ [21], and thus must involve a $b$ field. In conclusion, HSZ theory only has on-shell couplings of dilatons at two derivatives, and shares them with heterotic and bosonic strings. The latter two have a single on-shell coupling of the dilaton at four derivatives. These are indeed the predictions of the three factorized formulae.

\section{Derivative expansion of HSZ theory}

Our first goal is to give the action for $\mathcal{M}$ and $\phi$ in explicit form and organized by the number of derivatives, a number that can be zero, two, four, and six. While the parts with zero and two derivatives are known and take relatively simple forms [10, 21], the parts with four and six derivatives are considerably longer. We give their partially simplified forms and then their fully simplified forms when the dilaton field is set to zero. This will suffice for our later computation of on-shell three-point amplitudes for gravity and $b$ field fluctuations.

We will define actions $S$ as integrals over the double coordinates of the density $e^{\phi}$ times the Lagrangian $L$. For the theory in question [10] we have

$$
S=\int e^{\phi} L, \quad L=\frac{1}{2} \operatorname{tr}(\mathcal{T})-\frac{1}{6}\langle\mathcal{T} \mid \mathcal{T} \star \mathcal{T}\rangle .
$$

The field $\mathcal{T}$ is a tensor operator and encodes the double metric. For arbitrary tensor operators $T$ we have the expansion

$$
T=\frac{1}{2} T^{M N} Z_{M} Z_{N}-\frac{1}{2}\left(\hat{T}^{M} Z_{M}\right)^{\prime}
$$


here $T^{M N}$ and $\hat{T}^{M}$ are, respectively, the tensor part and the pseudo-vector part of the tensor operator. The trace of the tensor operator $T$ is a scalar operator $\operatorname{tr} T$ defined by (eq. (5.17), [10])

$$
\operatorname{tr} T \equiv \eta^{M N} T_{M N}-3\left(T^{M N} \partial_{M} \partial_{N} \phi+\partial \cdot \hat{T}+\hat{T} \cdot \partial \phi\right) .
$$

If a tensor operator $T$ is divergenceless, the pseudo-vector part is determined as a dilaton dependent function $G$ linear in the tensor component:

$$
\hat{T}^{M}=G^{M}\left(T_{P Q}\right)=G_{1}^{M}(T)+G_{3}^{M}(T),
$$

where $G_{1}$ and $G_{3}$ have one and three derivatives, respectively (eq. (5.37), [10]):

$$
\begin{aligned}
& G_{1}^{M}(T)=\partial_{N} T^{M N}+T^{M N} \partial_{N} \phi \\
& G_{3}^{M}(T)=-\frac{1}{2} T^{N P} \partial_{N} \partial_{P} \partial^{M} \phi-\frac{1}{2} \partial^{M}\left(\partial_{N} \partial_{P} T^{N P}+T^{N P}\left(\partial_{N} \partial_{P} \phi+\partial_{N} \phi \partial_{P} \phi\right)\right) .
\end{aligned}
$$

We make the following remarks:

1. The free index on $G_{3}$ is carried by a derivative.

2. $G_{1}(T)$ and $G_{3}(T)$ both vanish if the two indices in $T_{M N}$ are carried by derivatives,

3. $G_{3}(T)$ vanishes if one index on $T_{M N}$ is carried by a derivative.

The tensor operator $\mathcal{T}$ featuring in the action is parametrized by a double metric $\mathcal{M}^{M N}$, and the pseudo-vector part $\hat{\mathcal{M}}^{M}$ is determined by the condition that $\mathcal{T}$ is divergenceless:

$$
\mathcal{T}=\frac{1}{2} \mathcal{M}^{M N} Z_{M} Z_{N}-\frac{1}{2}\left(\hat{\mathcal{M}}^{N} Z_{M}\right)^{\prime}, \quad \hat{\mathcal{M}}^{M}=G^{M}(\mathcal{M}) .
$$

A short calculation gives

$\operatorname{tr} \mathcal{T}=\eta^{M N} \mathcal{M}_{M N}-3 \partial_{M} \partial_{N} \mathcal{M}^{M N}-6 \mathcal{M}^{M N} \partial_{M} \partial_{N} \phi-6 \partial_{M} \mathcal{M}^{M N} \partial_{N} \phi-3 \mathcal{M}^{M N} \partial_{M} \phi \partial_{N} \phi$,

which contains terms linear, quadratic and cubic in fields, and no more than two derivatives. We now use the star product $\star$ of two tensors, which gives a divergenceless tensor, to define

$$
W \equiv \mathcal{T} \star \mathcal{T}=\frac{1}{2} W^{M N} Z_{M} Z_{N}-\frac{1}{2}\left(\hat{W}^{M} Z_{M}\right)^{\prime},
$$

where the last equality defines the components of $W$. The definition of the star product ([10], section 6.2) implies that

$$
W^{M N} \equiv\left(\mathcal{T} \circ_{2} \mathcal{T}\right)^{M N}, \quad W^{M} \equiv G^{M}\left(W^{P Q}\right),
$$

the second following because $W$ is divergenceless. The formula for product $\mathrm{o}_{2}$ is given in (6.67) of [10]. ${ }^{3}$ The field $W^{M N}$ has an expansion on derivatives,

$$
W^{M N}=W_{0}^{M N}+W_{2}^{M N}+W_{4}^{M N}+W_{6}^{M N},
$$

\footnotetext{
${ }^{3}$ In [10] symmetrizations or antisymmetrizations carry no weight, in this paper they do.
} 
which, using the notation $\partial_{M_{1} \cdots M_{k}} \equiv \partial_{M_{1}} \cdots \partial_{M_{k}}$, takes the form

$$
\begin{aligned}
W_{0 M N}= & 2 \mathcal{M}_{M K} \mathcal{M}^{K}{ }_{N}, \\
W_{2 M N}= & -\frac{1}{2} \partial_{M} \mathcal{M}^{P Q} \partial_{N} \mathcal{M}_{P Q}+\mathcal{M}^{P Q} \partial_{P Q} \mathcal{M}_{M N}+4 \partial_{(M} \mathcal{M}^{K L} \partial_{L} \mathcal{M}_{N) K} \\
& -2 \partial_{Q} \mathcal{M}_{M}{ }^{P} \partial_{P} \mathcal{M}_{N}{ }^{Q}+G_{1}^{K}(\mathcal{M}) \partial_{K} \mathcal{M}_{M N}+2\left(\partial_{(N} G_{1}^{K}(\mathcal{M})-\partial^{K} G_{1(N}(\mathcal{M})\right) \mathcal{M}_{M) K}, \\
W_{4 M N}= & \partial_{M P} \mathcal{M}^{L K} \partial_{N L} \mathcal{M}_{K}{ }^{P}-2 \partial_{K(M} \mathcal{M}^{P Q} \partial_{P Q} \mathcal{M}_{N)}{ }^{K} \\
& +2\left(\partial_{(M} G_{3}^{K}(\mathcal{M})-\partial^{K} G_{3(M}(\mathcal{M})\right) \mathcal{M}_{N) K}-2 \partial_{P(M} G_{1}^{K}(\mathcal{M}) \partial_{K} \mathcal{M}^{P}{ }_{N)} \\
& +\partial_{P}\left(\partial_{(M} G_{1 Q}(\mathcal{M})-\partial_{Q} G_{1(M}(\mathcal{M})\right) \partial_{N)} \mathcal{M}^{P Q} \\
W_{6 M N}= & -\frac{1}{4} \partial_{M P Q} \mathcal{M}^{K L} \partial_{N K L} \mathcal{M}^{P Q}+\partial_{P}\left(\partial_{(M} G_{3 Q}(\mathcal{M})-\partial_{Q} G_{3(M}(\mathcal{M})\right) \partial_{N)} \mathcal{M}^{P Q} \\
& -\frac{1}{2} \partial_{P Q(M} G_{1}^{K}(\mathcal{M}) \partial_{N) K} \mathcal{M}^{P Q} .
\end{aligned}
$$

We note that

1. On $W_{4 M N}$ at least one index is carried by a derivative.

2. On $W_{6 M N}$ both indices are carried by derivatives.

We now turn to the pseudo-vector components $\hat{W}^{K}$ which, by definition are given by

$$
\hat{W}^{K}=G^{K}\left(W_{M N}\right)=G_{1}^{K}\left(W_{0}+W_{2}+W_{4}+W_{6}\right)+G_{3}^{K}\left(W_{0}+W_{2}+W_{4}+W_{6}\right) .
$$

It then follows by the remarks that the only terms in $W^{K}$ are:

$$
\begin{aligned}
& \hat{W}_{1}^{K}=G_{1}^{K}\left(W_{0}\right), \\
& \hat{W}_{3}^{K}=G_{1}^{K}\left(W_{2}\right)+G_{3}^{K}\left(W_{0}\right), \\
& \hat{W}_{5}^{K}=G_{1}^{K}\left(W_{4}\right)+G_{3}^{K}\left(W_{2}\right) .
\end{aligned}
$$

These are terms with one, three, and five derivatives. Note that on $G_{1}\left(W_{4}\right)$ the free index is on a derivative because it is an index on $W_{4}$ and the other index on $W_{4}$ must be the non-derivative one to have a non vanishing contribution. Thus the free index in $\hat{W}_{5}$ is on a derivative.

It is now possible to evaluate the full Lagrangian in (3.1). For the cubic term we need the inner product formula that follows from eq. (6.67) of [10]

$$
\begin{aligned}
\left\langle T_{1} \mid T_{2}\right\rangle= & \frac{1}{2} T_{1}^{P Q} T_{2 P Q}-\partial_{P} T_{1}^{K L} \partial_{L} T_{2 K}{ }^{P}+\frac{1}{4} \partial_{P Q} T_{1}^{K L} \partial_{K L} T_{2}^{P Q} \\
& -\frac{3}{2}\left(\hat{T}_{1}^{M} \hat{T}_{2}^{N} \eta_{M N}-\partial_{N} \hat{T}_{1}^{M} \partial_{M} \hat{T}_{1}^{N}\right)-\frac{3}{2}\left(\partial_{P} \hat{T}_{1}^{K} T_{2 K}{ }^{P}+\partial_{P} \hat{T}_{2}^{K} T_{1 K}{ }^{P}\right) \\
& +\frac{3}{4}\left(\partial_{P Q} \hat{T}_{1}^{K} \partial_{K} T_{2}^{P Q}+\partial_{P Q} \hat{T}_{2}^{K} \partial_{K} T_{1}^{P Q}\right) .
\end{aligned}
$$

This formula must be used for $T_{1}=\mathcal{T}$ and $T_{2}=W$. A useful identity, easily derived by integration by parts, reads

$$
\int e^{\phi} f^{K} G_{(1) K}(T)=\int e^{\phi}\left(-\partial^{P} f^{K} T_{K P}\right) .
$$


Using this identity and the earlier results we find the following terms in the Lagrangian

$$
\begin{aligned}
L_{0}= & -\frac{1}{6} \mathcal{M}_{M N} \mathcal{M}^{N P} \mathcal{M}_{P}^{M}+\frac{1}{2} \mathcal{M}_{M}^{M} \\
L_{2}= & \frac{1}{2}\left(\mathcal{M}^{2}-1\right)^{M P} \mathcal{M}_{P}{ }^{N} \partial_{M} \partial_{N} \phi+\frac{1}{8} \mathcal{M}^{M N} \partial_{M} \mathcal{M}^{P Q} \partial_{N} \mathcal{M}_{P Q} \\
& -\frac{1}{2} \mathcal{M}^{M N} \partial_{N} \mathcal{M}^{K L} \partial_{L} \mathcal{M}_{K M}-\mathcal{M}^{M N} \partial_{M} \partial_{N} \phi \\
L_{4}= & -\frac{1}{12} \mathcal{M}^{M N} W_{4 M N}+\frac{1}{6} \partial^{P} \mathcal{M}^{K L} \partial_{L} W_{2 K P}+\frac{1}{4} \partial_{P} G_{1}^{K}\left(W_{2}\right) \mathcal{M}_{K}^{P} \\
& -\frac{1}{24} \partial_{P} \partial_{Q} \mathcal{M}^{K L} \partial_{K} \partial_{L} W_{0}^{P Q} \\
& -\frac{1}{4} \partial_{N} G_{1}^{M}(\mathcal{M}) \partial_{M} G_{1}^{N}\left(W_{0}\right)-\frac{1}{8} \partial_{P} \partial_{Q} G_{1}^{K}(\mathcal{M}) \partial_{K} W_{0}^{P Q}-\frac{1}{8} \partial_{P} \partial_{Q} G_{1}^{K}\left(W_{0}\right) \partial_{K} \mathcal{M}^{P Q}, \\
L_{6}= & -\frac{1}{12} \mathcal{M}^{M N} W_{6 M N}+\frac{1}{6} \partial^{P} \mathcal{M}^{K L} \partial_{L} W_{4 K P}+\frac{1}{4} \partial_{P} G_{1}^{K}\left(W_{4}\right) \mathcal{M}_{K}^{P} \\
& -\frac{1}{24} \partial_{P} \partial_{Q} \mathcal{M}{ }^{K L} \partial_{K} \partial_{L} W_{(2)}^{P Q} \\
& -\frac{1}{4} \partial_{N} G_{1}^{M}(\mathcal{M}) \partial_{M} G_{1}^{N}\left(W_{2}\right)-\frac{1}{8} \partial_{P} \partial_{Q} G_{1}^{K}(\mathcal{M}) \partial_{K} W_{2}^{P Q}-\frac{1}{8} \partial_{P} \partial_{Q} G_{1}^{K}\left(W_{2}\right) \partial_{K} \mathcal{M}^{P Q}
\end{aligned}
$$

The results for the zero and two derivative part of the Lagrangian were given in $[10,21]$ and cannot be simplified further. One can quickly show that the last line of $L_{4}$ and $L_{6}$ vanish if we have zero dilaton derivatives. Also the last two terms in the first lines of $L_{4}$ and $L_{6}$ admit simplification. Still keeping all terms, we can simplify $L_{4}$ and $L_{6}$ to read

$$
\begin{aligned}
L_{4}= & -\frac{1}{12} \mathcal{M}^{M N} W_{4 M N}+\frac{1}{12} \partial^{M} G_{1}^{N}(\mathcal{M}) W_{2 M N}+\frac{1}{6} \mathcal{M}^{M K} W_{2 K}{ }^{N} \partial_{M N} \phi \\
& -\frac{1}{24} \partial_{P} \partial_{Q} \mathcal{M}^{K L} \partial_{K} \partial_{L} W_{0}^{P Q} \\
& +\frac{1}{4} G_{1}^{M}(\mathcal{M}) G_{1}^{N}\left(W_{0}\right) \partial_{M N} \phi+\frac{1}{8}\left(G_{1}^{K}(\mathcal{M}) W_{0}^{P Q}+G_{1}^{K}\left(W_{0}\right) \mathcal{M}^{P Q}\right) \partial_{K P Q} \phi, \\
L_{6}= & -\frac{1}{12} \mathcal{M}^{M N} W_{6 M N}+\frac{1}{12} \partial^{M} G_{1}^{N}(\mathcal{M}) W_{4 M N}+\frac{1}{6} \mathcal{M}^{M K} W_{4 K}{ }^{N} \partial_{M N} \phi \\
& -\frac{1}{24} \partial_{P} \partial_{Q} \mathcal{M}^{K L} \partial_{K} \partial_{L} W_{2}^{P Q} \\
& +\frac{1}{4} G_{1}^{M}(\mathcal{M}) G_{1}^{N}\left(W_{2}\right) \partial_{M N} \phi+\frac{1}{8}\left(G_{1}^{K}(\mathcal{M}) W_{2}^{P Q}+G_{1}^{K}\left(W_{2}\right) \mathcal{M}^{P Q}\right) \partial_{K P Q} \phi .
\end{aligned}
$$

The fourth and sixth derivative part of the Lagrangian, written explicitly in terms of $\mathcal{M}$ and $\phi$ are rather long. Since we will focus in this paper on gravity and $b$ field three-point amplitudes, we will ignore the dilaton. With dilaton fields set to zero a computation gives:

$$
\begin{aligned}
\left.L_{4}\right|_{\phi=0}= & \mathcal{M}^{M N}\left(\frac{1}{6} \partial_{M L} \mathcal{M}^{P Q} \partial_{P Q} \mathcal{M}_{N}{ }^{L}-\frac{1}{12} \partial_{N P} \mathcal{M}^{L Q} \partial_{M L} \mathcal{M}^{P}{ }_{Q}\right. \\
& +\frac{1}{12} \partial_{M N} \mathcal{M}_{K Q} \partial_{P}^{K} \mathcal{M}^{P Q}-\frac{1}{12} \partial_{M P} \mathcal{M}^{P Q} \partial_{N K} \mathcal{M}_{Q}{ }^{K} \\
& \left.+\frac{1}{3} \partial_{P} \mathcal{M}_{M}{ }^{K} \partial_{N K Q} \mathcal{M}^{P Q}-\frac{1}{6} \partial_{M} \mathcal{M}^{P Q} \partial_{P K[N} \mathcal{M}_{Q]}{ }^{K}\right) \\
& +\partial^{M P} \mathcal{M}_{P}{ }^{N}\left(\frac{1}{6} \partial_{N} \mathcal{M}^{K L} \partial_{L} \mathcal{M}_{M K}-\frac{1}{6} \partial_{Q} \mathcal{M}_{M}{ }^{K} \partial_{K} \mathcal{M}_{N}{ }^{Q}+\frac{1}{12} \partial_{L} \mathcal{M}^{K L} \partial_{K} \mathcal{M}_{M N}\right)
\end{aligned}
$$




$$
\begin{aligned}
\left.L_{6}\right|_{\phi=0}= & \mathcal{M}^{M N}\left(\frac{1}{48} \partial_{M P Q} \mathcal{M}^{K L} \partial_{N K L} \mathcal{M}^{P Q}+\frac{1}{24} \partial_{M P Q L} \mathcal{M}^{K L} \partial_{N K} \mathcal{M}^{P Q}\right. \\
& \left.-\frac{1}{24} \partial_{P Q K L} \mathcal{M}^{K L} \partial_{M N} \mathcal{M}^{P Q}+\frac{1}{12} \partial_{M P K L} \mathcal{M}^{K L} \partial_{N Q} \mathcal{M}^{P Q}\right) \\
& -\frac{1}{24} \partial_{M N K L} \mathcal{M}^{K L}\left(\partial_{P} \mathcal{M}^{P Q} \partial_{Q} \mathcal{M}^{M N}-2 \partial_{P} \mathcal{M}^{M Q} \partial_{Q} \mathcal{M}^{N P}\right) \\
& -\frac{1}{24} \partial_{N L} \mathcal{M}^{M L}\left(2 \partial_{M K} \mathcal{M}^{P Q} \partial_{P Q} \mathcal{M}_{N}{ }^{K}+2 \partial_{M P Q} \mathcal{M}^{K Q} \partial_{K} \mathcal{M}^{P}{ }_{N}+\partial_{P Q R} \mathcal{M}^{N R} \partial_{M} \mathcal{M}^{P Q}\right) .
\end{aligned}
$$

\section{Perturbative expansion of HSZ theory}

In this section we discuss the perturbative expansion of the Lagrangian obtained in the previous section around a constant background $\langle\mathcal{M}\rangle$ that can be identified with a constant generalized metric, as discussed in [21]. We define projected $O(D, D)$ indices as follows:

$$
V_{\underline{M}}=P_{M}^{N} V_{N}, \quad V_{\bar{M}}=\bar{P}_{M}^{N} V_{N},
$$

where the projectors are defined as:

$$
P_{M}^{N}=\frac{1}{2}(\eta-\overline{\mathcal{H}})_{M}{ }^{N}, \quad \bar{P}_{M}{ }^{N}=\frac{1}{2}(\eta+\overline{\mathcal{H}})_{M^{N}} .
$$

Here $\overline{\mathcal{H}}$ is the background, constant, generalized metric. We expand the double metric $\mathcal{M}$ as follows:

$$
\mathcal{M}_{M N}=\overline{\mathcal{H}}_{M N}+m_{M N}=\overline{\mathcal{H}}_{M N}+m_{\underline{M} \underline{N}}+m_{\underline{M} \bar{N}}+m_{\bar{M} \underline{N}}+m_{\bar{M} \bar{N}},
$$

where we have decomposed the fluctuations $m_{M N}$ into projected indices. It was shown in [21] that the projections $m_{\bar{M} \bar{N}}$ and $m_{\underline{M}}$ are auxiliary fields and the physical part of the metric and the $b$-field fluctuations are encoded in $m_{\underline{\underline{M}} \bar{N}}=m_{\bar{N} \underline{\underline{M}}}$. To obtain the Lagrangian in terms of physical fields, we need to expand it in fluctuations and then eliminate the auxiliary fields using their equations of motion. To illustrate this procedure more clearly, and for ease of readability we will write

$$
a_{\underline{M} \underline{N}} \equiv m_{\underline{M} \underline{N}}, \quad a_{\bar{M} \bar{N}} \equiv m_{\bar{M} \bar{N}},
$$

where the label $a$ for the field reminds us that it is auxiliary. With this notation the $\mathcal{M}$ field expansion reads

$$
\mathcal{M}_{M N}=\overline{\mathcal{H}}_{M N}+a_{\underline{M} \underline{N}}+m_{\underline{M} \bar{N}}+m_{\bar{M} \underline{N}}+a_{\bar{M} \bar{N}} .
$$

Let us now carry out the procedure of elimination of auxiliary field explicitly for the two derivative part of the Lagrangian.

\subsection{Perturbative expansion of the two-derivative Lagrangian}

Let us use $L^{(i, j)}$ to denote the part of the Lagrangian with $i$ fields and $j$ derivatives. In what follows, we are only interested in the Lagrangian up to cubic order in fields, so we will ignore all terms with more than three fields. Also note that the Langrangian appears 
in the action multiplied with a factor of $e^{\phi}$. Using the expansion (4.5) we see that the zero derivative Lagrangian $L_{0}$ has terms quadratic and cubic in field fluctuations:

$$
e^{\phi} L_{0}=L^{(2,0)}+L^{(3,0)}+\cdots
$$

where the dots denote terms quartic in fields and

$$
\begin{aligned}
L^{(2,0)}= & \frac{1}{2} a^{\underline{M} \underline{N}} a_{\underline{M} \underline{N}}-\frac{1}{2} a^{\bar{M} \bar{N}} a_{\bar{M} \bar{N}}, \\
L^{(3,0)}= & -\frac{1}{2} a^{\underline{M}} \underline{\underline{N}} m_{\underline{M}}{ }^{\bar{P}} m_{\underline{N} \bar{P}}-\frac{1}{6} a^{\underline{M}} \underline{\underline{N}} a_{\underline{\underline{M}}} a_{\underline{\underline{N}} \underline{\underline{P}}}-\frac{1}{2} a^{\bar{M} \bar{N}} m^{\underline{P}} \bar{M}_{\underline{\underline{P}} \bar{N}}-\frac{1}{6} a^{\bar{M} \bar{N}} a_{\bar{N}} a_{\bar{N}} a_{\bar{P}} \\
& +\frac{1}{2} \phi\left(a^{\underline{M}} \underline{\underline{N}} a_{\underline{M} \underline{N}}-a^{\bar{M} \bar{N}} a_{\bar{M} \bar{N}}\right) .
\end{aligned}
$$

If we denote generically by $a$ an auxiliary field $\left(a_{\underline{M} \underline{N}}\right.$ or $\left.a_{\bar{M}} \bar{N}\right)$ and by $m$ the physical field $m_{\underline{M} \bar{N}}$, the structure of terms with auxiliary field that we find here is

$$
a^{2}+a m^{2}+a^{3}+a^{2} m .
$$

If we solve for the auxiliary field based on the above, to leading order we will find $a \sim m^{2}$. The perturbative expansion for the two-derivative Lagrangian $L_{2}$ in (3.16) is more involved. It decomposes into a quadratic and a cubic part in fluctuations:

$$
e^{\phi} L_{2}=L^{(2,2)}+L^{(3,2)}+\cdots
$$

and we find

$$
\begin{aligned}
& L^{(2,2)}=\frac{1}{2} \partial^{\bar{M}} m^{\underline{P} \bar{Q}} \partial_{\bar{M}} m_{\underline{P} \bar{Q}}+\frac{1}{2} \partial^{\underline{M}} m^{\underline{P} \bar{Q}} \partial_{\underline{P}} m_{\underline{M} \bar{Q}}-\frac{1}{2} \partial^{\bar{M}} m^{\underline{P} \bar{Q}} \partial_{\bar{Q}} m_{\underline{P} \bar{M}} \\
& -2 m^{\underline{M} \bar{N}} \partial_{\underline{M}} \partial_{\bar{N}} \phi-2 \phi \partial^{\bar{M}} \partial_{\bar{M}} \phi \\
& +\frac{1}{4} \partial^{\bar{M}} a^{\bar{P} \bar{Q}} \partial_{\bar{M}} a_{\bar{P} \bar{Q}}+\frac{1}{4} \partial^{\bar{M}} a^{\underline{P} \underline{Q}} \partial_{\bar{M}} a_{\underline{P} Q} \\
& +\frac{1}{2} \partial^{\underline{M}} a^{\underline{\underline{P}}} \partial_{\underline{Q}} a_{\underline{P} \underline{M}}-\frac{1}{2} \partial^{\bar{M}} a^{\bar{P} \bar{Q}} \partial_{\bar{Q}} a_{\bar{P} \bar{M}}, \\
& L^{(3,2)}=\frac{1}{2} m^{\underline{M} \bar{N}}\left(\partial_{\underline{M}} m^{\underline{P} \bar{Q}} \partial_{\bar{N}} m_{\underline{P} \bar{Q}}-\partial_{\underline{M}} m^{\underline{P} \bar{Q}} \partial_{\bar{Q}} m_{\underline{P} \bar{N}}-\partial_{\bar{N}} m^{\underline{P} \bar{Q}} \partial_{\underline{P}} m_{\underline{M} \bar{Q}}\right) \\
& +\frac{1}{2} \phi\left(\partial_{\bar{M}} m^{\underline{P} \bar{Q}} \partial^{\bar{M}} m_{\underline{P} \bar{Q}}-\partial_{\bar{M}} m^{\underline{P}} \bar{Q} \partial_{\bar{Q}} m_{\underline{P}}^{\bar{M}}+\partial^{\underline{M}} m^{\underline{P} \bar{Q}} \partial_{\underline{P}} m_{\underline{M} \bar{Q}}\right) \\
& -\frac{1}{2}\left(m_{\underline{M}}^{\bar{P}} m_{\underline{N} \bar{P}} \partial^{\underline{M}} \partial^{\underline{N}} \phi-m^{\underline{P}} \bar{M} m_{\underline{P} \bar{N}} \partial^{\bar{M}} \partial^{\bar{N}} \phi\right) \\
& -\phi^{2} \partial_{\bar{M}} \partial^{\bar{M}} \phi-2 \phi m^{\underline{M}} \bar{N} \partial_{\underline{M}} \partial_{\bar{N}} \phi+L_{\text {aux }}^{(3,2)} \text {, }
\end{aligned}
$$

where $L_{\text {aux }}^{(3,2)}$ denotes the terms that contain at least one auxiliary field. The precise expression for these terms will not be needed. Note, however, from $L^{(2,2)}$ that we have terms of the form

$$
\partial а \partial а
$$

and from $L^{(3,2)}$ terms that couple an a field to two fields in a term with two derivatives. 
Next, we eliminate the auxiliary fields from the total Lagrangian with three or less fields and at most two derivatives.

$$
L^{(\leq 3,2)}=L^{(2,0)}+L^{(3,0)}+L^{(2,2)}+L^{(3,2)},
$$

From the terms in (4.7) and (4.10), denoted schematically in (4.8) and (4.11), we now find:

$$
a_{\bar{M} \bar{N}}=-\frac{1}{2} m^{\underline{P}}{ }_{\bar{M}} m_{\underline{P} \bar{N}}+\cdots, \quad a_{\underline{M} \underline{N}}=\frac{1}{2} m_{\underline{M}}{ }^{\bar{P}} m_{\underline{N} \bar{P}}+\cdots .
$$

where the dots denote terms with at least two fields and at least two derivatives. Now, we plug this solution for the auxiliary field into the Lagrangian $L^{(\leq 3,2)}$ and keep only terms with two derivatives and up to cubic order in physical fields. The terms indicated by dots in (4.13) do not contribute; they always lead to terms with at least four fields or at least four derivatives. Nor does $L_{\text {aux }}^{(3,2)}$ lead to any contributions. In fact, most of the terms involving auxiliary fields do not contribute. After a short computation, we obtain the following two derivative Lagrangian completely in terms of the physical fields:

$$
\begin{aligned}
& L^{(\leq 3,2)}=\frac{1}{2} \partial^{\bar{M}} m^{\underline{P} \bar{Q}} \partial_{\bar{M}} m_{\underline{P} \bar{Q}}+\frac{1}{2} \partial^{\underline{M}} m^{\underline{P} \bar{Q}} \partial_{\underline{P}} m_{\underline{M} \bar{Q}}-\frac{1}{2} \partial^{\bar{M}} m^{\underline{P} \bar{Q}} \partial_{\bar{Q}} m_{\underline{P} \bar{M}} \\
& -2 m^{\underline{M}} \bar{N} \partial_{\underline{M}} \partial_{\bar{N}} \phi-2 \phi \partial^{\bar{M}} \partial_{\bar{M}} \phi \\
& +\frac{1}{2} m^{\underline{M} \bar{N}}\left(\partial_{\underline{M}} m^{\underline{P} \bar{Q}} \partial_{\bar{N}} m_{\underline{P} \bar{Q}}-\partial_{\underline{M}} m^{\underline{P} \bar{Q}} \partial_{\bar{Q}} m_{\underline{P} \bar{N}}-\partial_{\bar{N}} m^{\underline{P} \bar{Q}} \partial_{\underline{P}} m_{\underline{M} \bar{Q}}\right) \\
& +\frac{1}{2} \phi\left(\partial_{\bar{M}} m^{\underline{P} \bar{Q}} \partial^{\bar{M}} m_{\underline{P} \bar{Q}}-\partial_{\bar{M}} m^{\underline{P} \bar{Q}} \partial_{\bar{Q}} m_{\underline{P}}^{\bar{M}}+\partial^{\underline{M}} m^{\underline{P} \bar{Q}} \partial_{\underline{P}} m_{\underline{M} \bar{Q}}\right) \\
& -\frac{1}{2}\left(m_{\underline{M}}{ }^{\bar{P}} m_{\underline{N} \bar{P}} \partial^{\underline{M}} \partial^{\underline{N}} \phi-m^{\underline{P}} \bar{M}_{\underline{P}} m_{\bar{N}} \partial^{\bar{M}} \partial^{\bar{N}} \phi\right) \\
& -\phi^{2} \partial_{\bar{M}} \partial^{\bar{M}} \phi-2 \phi m^{\underline{M}} \bar{N} \partial_{\underline{M}} \partial_{\bar{N}} \phi \text {. }
\end{aligned}
$$

Next, we write the action in terms of double field theory (or string field theory) variables $e_{i j}$. The way to translate from $m_{\underline{M} \bar{N}}$ variables to $e_{i j}$ variables is explained in section 5.3 of [21]. Here is the rule that follows: convert all barred and under-barred indices into latin indices respecting the contractions, replacing $m$ by $e$, underbar derivatives by $D$ and barred derivatives by $\bar{D}$, and multiply by a coefficient that is the product of a factor of 2 for each $m$ field, a factor of $+\frac{1}{2}$ for each barred contraction, and a factor of $-\frac{1}{2}$ for each under-barred contraction. As an example, consider the second term on the first line of (4.14), after integration by parts, it becomes:

$\frac{1}{2} \partial^{\underline{M}} m^{\underline{P} \bar{Q}} \partial_{\underline{P}} m_{\underline{M} \bar{Q}}=\frac{1}{2} \partial_{\underline{P}} m^{\underline{P} \bar{Q}} \partial^{\underline{M}} m_{\underline{M} \bar{Q}} \quad \rightarrow \frac{1}{2} \cdot 2^{2} \cdot \frac{1}{2}\left(-\frac{1}{2}\right)^{2} D_{p} e^{p q} D^{m} e_{m q}=\frac{1}{4} D_{p} e^{p q} D^{m} e_{m q}$.

Using this technique for all the terms appearing in the Lagrangian (4.14) we obtain:

$$
\begin{aligned}
L^{(\leq 3,2)}= & \frac{1}{4}\left(e^{i j} \bar{D}^{2} e_{i j}+\left(D^{i} e_{i j}\right)^{2}+\left(\bar{D}^{i} e_{i j}\right)^{2}\right)+e^{i j} D_{i} \bar{D}_{j} \phi-\phi \bar{D}^{2} \phi . \\
& +\frac{1}{4} e_{i j}\left(D^{i} e_{k l} \bar{D}^{j} e^{k l}-D^{i} e_{k l} \bar{D}^{l} e^{k j}-D^{k} e^{i l} \bar{D}^{j} e_{k l}\right) \\
& -\frac{1}{4} \phi\left(\left(D^{i} e_{i j}\right)^{2}+\left(\bar{D}^{j} e_{i j}\right)^{2}+\frac{1}{2}\left(D_{k} e_{i j}\right)^{2}+\frac{1}{2}\left(\bar{D}_{k} e_{i j}\right)^{2}+2 e^{i j}\left(D_{i} D^{k} e_{k j}+\bar{D}_{j} \bar{D}^{k} e_{i k}\right)\right) \\
& +\phi e_{i j} D^{i} \bar{D}^{j} \phi-\frac{1}{2} \phi^{2} \bar{D}^{2} \phi .
\end{aligned}
$$


With the identification $\phi=-2 d$ the above cubic Lagrangian becomes precisely the double field theory Lagrangian in equation (3.25) of [7]. From the quadratic part of the above action, we see that the kinetic term of $\phi$ has wrong sign. This is, because the action (4.16) is in the string frame and $\phi$ is not the physical dilaton. To obtain the action in terms of physical fields $\hat{e}_{i j}$ and $\hat{\phi}$ that decouple at the quadratic level, we need a field re-definition. Physical fields $\hat{e}_{i j}$ and $\hat{\phi}$ are obtained in the Einstein frame as a linear combination of $e_{i j}$ and $\phi$. We write schematically:

$$
e_{i j} \sim \hat{e}_{i j}+\hat{\phi} \eta_{i j}, \quad \phi \sim \hat{\phi}+\hat{e}_{i}{ }^{i}
$$

If we are looking for pure gravitational three-point amplitudes the first redefinition need not be performed in the action, as it would give rise to terms that involve the dilaton. The second one is not needed either, since on-shell gravitons have traceless polarizations.

After solving the strong constraint by setting $\tilde{\partial}^{i}=0$ and setting the dilaton to zero, the above Lagrangian becomes:

$$
\left.L^{(\leq 3,2)}\right|_{\phi=0}=\frac{1}{4}\left(e^{i j} \partial^{2} e_{i j}+2\left(\partial^{i} e_{i j}\right)^{2}\right)+\frac{1}{4} e_{i j}\left(\partial^{i} e_{k l} \partial^{j} e^{k l}-\partial^{i} e_{k l} \partial^{l} e^{k j}-\partial^{k} e^{i l} \partial^{j} e_{k l}\right) .
$$

For an off-shell three-point vertex all terms in the cubic Lagrangian must be kept. But for the computation of on-shell three-point amplitudes we may use the on-shell conditions to simplify the cubic Lagrangian. These conditions can be stated as follows in terms of $e_{i j}$.

$$
\partial^{i} e_{i j}=\partial^{j} e_{i j}=0, \quad \partial_{i} e^{*} \partial^{i} e^{* \cdots} \cdots=0 .
$$

The first condition is transversality and the second condition follows from the momentum conservation and masslessness. For the cubic terms in (4.18) the on-shell conditions do not lead to any further simplification and we record:

$$
\left.L^{(3,2)}\right|_{\phi=0, \text { on-shell }}=\frac{1}{4} e_{i j}\left(\partial^{i} e_{k l} \partial^{j} e^{k l}-\partial^{i} e_{k l} \partial^{l} e^{k j}-\partial^{p} e^{i q} \partial^{j} e_{p q}\right) .
$$

Three-point on-shell amplitudes can now be computed from this expression.

\subsection{General treatment of auxiliary fields}

Here we argue that for the purposes of three-point on-shell amplitudes and, with the dilaton set to zero, the auxiliary field does not affect the Lagrangian and can safely be ignored. To prove the claim we must use on-shell conditions (4.19): we will argue that any contribution from auxiliary fields vanishes upon use of these conditions. It is straightforward to translate these on-shell conditions in terms of the double metric fluctuations. They can be written as:

$$
\partial_{\underline{M}} m^{\underline{M}} \bar{N}=\partial_{\bar{N}} m^{\underline{M} \bar{N}}=\partial_{\bar{M}} m^{\cdot} \partial^{\bar{M}} m^{*} \cdots=0 .
$$

Setting all dilatons to zero, the only physical field is $m_{M \bar{N}}$, which we symbolically represent by $m$. The most general form of the Lagrangian involving at least one auxiliary field is as follows:

$$
L[a, m]=a m+a^{2}+a^{3}+a^{2} m+a m^{2} .
$$


Since the theory is cubic in $\mathcal{M}$ and the dilaton is set to zero, this is all there is. In here we are leaving derivatives implicit; all the above terms can carry up-to six derivatives. As we have seen before, there is no am coupling with zero derivatives nor with two derivatives. Let us now see that no such term exists that does not vanish using the on-shell conditions. The general term of this kind would be

$$
m_{\bar{M} \bar{N}}\left(\cdots m_{\underline{P} \bar{Q}}\right)
$$

where the dots represent derivatives or metrics $\eta$ that contract same type indices, barred or un-barred. These are required to contract all indices and yield an $O(D, D)$ invariant. Since integration by parts is allowed we have assumed, without loss of generality that all derivatives are acting on the physical field. Since the un-barred index $P$ is the only unbarred index, it must be contracted with a derivative. Thus the term must be of the form

$$
m_{\bar{M} \bar{N}}\left(\cdots \partial^{\underline{P}} m_{\underline{P} \bar{Q}}\right) .
$$

Regardless of what we do to deal with the other barred indices, we already see that this coupling vanishes using the on-shell conditions, proving the claim.

The Lagrangian (4.22) then reduces to the following:

$$
L[a, m]=a^{2}+a^{3}+a^{2} m+a m^{2} .
$$

The equation of motion for the auxiliary field is, schematically, $a \sim m^{2}+a m+a^{2}$, which implies that a perturbative solution in powers of physical fields begins with terms quadratic on the physical fields. Thus we write

$$
a(m)=a_{2}(m)+a_{3}(m)+\cdots,
$$

where dots indicate terms with quartic or higher powers of $m$. But now it is clear that substitution back into (4.25) can only lead to terms with quartic or higher powers of $m$. This concludes our argument that the elimination of auxiliary fields is not required for the computation of on-shell three-point amplitudes for metric and $b$ fields.

\subsection{Higher-derivative Lagrangian and on-shell amplitudes}

In this subsection we perform the perturbative expansion of the four and six derivative Lagrangian and compute the on-shell three-point amplitudes. We use the on-shell conditions (4.21) and ignore the auxiliary field in light of our earlier discussion. We note that

$$
\begin{aligned}
& \partial^{M} \mathcal{M}_{M \bar{N}}=\partial^{\bar{M}} a_{\bar{M} \bar{N}}+\partial^{\underline{M}} m_{\underline{M} \bar{N}}, \\
& \partial^{M} \mathcal{M}_{M \underline{N}}=\partial^{\bar{M}} m_{\bar{M} \underline{N}}+\partial^{\underline{M}} a_{\underline{M} \underline{N}} .
\end{aligned}
$$

Since we are allowed to set auxiliary fields to zero and to use the on-shell conditions (4.21), both $\partial^{M} \mathcal{M}_{M \bar{N}}$ and $\partial^{M} \mathcal{M}_{M N}$ can be set to zero, and as a result, we are allowed to set

$$
\partial^{M} \mathcal{M}_{M N} \rightarrow 0
$$

in simplifying the higher-derivative cubic interactions! This is a great simplification. 
Now we use (4.28) in the four derivative Lagrangian $L_{4}$ given in (3.18). Only the terms on the first line survive and we get:

$$
\left.L_{4}\right|_{\phi=0}=\frac{1}{6} \mathcal{M}^{M N} \partial_{N L} \mathcal{M}^{P Q} \partial_{P Q} \mathcal{M}_{M}^{L}-\frac{1}{12} \mathcal{M}^{M N} \partial_{N P} \mathcal{M}^{L Q} \partial_{M L} \mathcal{M}^{P}{ }_{Q}
$$

Now we plug in the expansion (4.5) and keep only the cubic terms which do not vanish on-shell. After a short computation we obtain the four derivative cubic Lagrangian in terms of the physical fields

$$
\left.L^{(3,4)}\right|_{\substack{\phi=0 \\ \text { on-shell }}}=\frac{1}{3} m^{\underline{M} \bar{N}}\left(\partial_{\bar{N} \bar{L}} m^{\underline{P} \bar{Q}}\left[\partial_{\underline{P} \bar{Q}} m_{\underline{M}}^{\bar{L}}-\frac{1}{2} \partial_{\underline{M} \bar{Q}} m_{\underline{P}}{ }^{\bar{L}}\right]+\partial_{\underline{M} \underline{L}} m^{\underline{P} \bar{Q}}\left[\partial_{\underline{P} \bar{Q}} m^{\underline{L}} \bar{N}-\frac{1}{2} \partial_{\bar{N} \underline{P}} m^{\underline{L}} \bar{Q}\right]\right) .
$$

Translating this to $e$ fluctuations (three $m$ 's and 5 contractions):

$$
\left.L^{(3,4)}\right|_{\substack{\phi=0 \\ \text { on-shell }}}=\frac{1}{12} e^{i j}\left(\partial_{j l} e^{p q}\left[\partial_{p q} e_{i}^{l}-\frac{1}{2} \partial_{i q} e_{p}^{l}\right]-\partial_{i l} e^{p q}\left[\partial_{p q} e_{j}^{l}-\frac{1}{2} \partial_{p j} e_{q}^{l}\right]\right) .
$$

Using integration by parts and the gauge conditions this simplifies into:

$$
\left.L^{(3,4)}\right|_{\substack{\phi=0 \\ \text { on-shell }}}=\frac{1}{8} e_{i j}\left(\partial^{j q} e_{k l} \partial^{k l} e_{q}^{i}-\partial^{i p} e_{k l} \partial^{k l} e_{p}{ }^{j}\right)
$$

and written in terms of the metric and $b$ field fluctuations using $e_{i j}=h_{i j}+b_{i j}$ :

$$
\left.L^{(3,4)}\right|_{\substack{\phi=0 \\ \text { on-shell }}}=\frac{1}{2} b^{i j} \partial_{j l} h^{m n} \partial_{m n} h_{i}^{l} .
$$

A short computation confirms that this result is precisely produced by the on-shell perturbative evaluation of the action

$$
L^{(3,4)}=-\frac{1}{2} H^{i j k} \Gamma_{i p}^{q} \partial_{j} \Gamma_{k q}^{p},
$$

given in equation (3.23) of [11] and arising from the expansion of the kinetic term for the Chern-Simons improved field strength $\widehat{H}$. There is no Riemann-squared term appearing, as has been argued before.

In the six-derivative Lagrangian $L_{6}$ given in (3.18) only the first term survives after we impose the on-shell condition. Integrating by parts the $\partial_{N}$ derivative we have

$$
\left.L_{6}\right|_{\substack{\phi=0 \\ \text { on-shell }}}=-\frac{1}{48} \mathcal{M}^{M N} \partial_{M N P Q} \mathcal{M}^{K L} \partial_{K L} \mathcal{M}^{P Q}
$$

Using the $\mathcal{M}$ field expansion and keeping only cubic terms which are non-vanishing on-shell, we get:

$$
\left.L^{(3,6)}\right|_{\substack{\phi=0 \\ \text { on-shell }}}=-\frac{1}{6} m^{\underline{M} \bar{N}} \partial_{\underline{M}} \bar{N} \underline{P} \bar{Q}^{\underline{K}} m^{\underline{L}} \partial_{\underline{K} \bar{L}} m^{\underline{P} \bar{Q}} .
$$

In term of $e_{i j}$ this takes the form:

$$
\left.L^{(3,6)}\right|_{\substack{\phi=0 \\ \text { on-shell }}}=\frac{1}{48} e_{i j} \partial^{i j p q} e_{k l} \partial^{k l} e_{p q} .
$$


The structure of the six-derivative term is such that only the symmetric part of $e_{i j}$ contributes. In terms of the metric fluctuations we get:

$$
\left.L^{(3,6)}\right|_{\substack{\phi=0 \\ \text { on-shell }}}=\frac{1}{48} h_{i j} \partial^{i j p q} h_{k l} \partial^{k l} h_{p q} .
$$

This term is produced by the perturbative on-shell evaluation of the following Riemanncubed term:

$$
-\frac{1}{48} R_{i j}{ }^{k l} R_{k l}^{p q} R_{p q}{ }^{i j}
$$

where the linearized Riemann tensor is: $R_{i j k l}=\frac{1}{2}\left(\partial_{j k} h_{i l}+\partial_{i l} h_{j k}-\partial_{k i} h_{j l}-\partial_{j l} h_{i k}\right)$. A short computation then shows:

$$
-\left.\frac{1}{48} R_{i j}{ }^{k l} R_{k l}^{p q} R_{p q}{ }^{i j}\right|_{\text {on-shell }}=-\frac{1}{48} \partial^{l q} h_{i j} \partial^{p j} h_{k l} \partial^{k i} h_{p q},
$$

which gives precisely the term (4.38) after integration by parts.

Collecting our results (4.20), (4.32) and (4.37) for the cubic interactions with two, four, and six derivatives, we have:

$$
\begin{aligned}
\left.L_{3}\right|_{\substack{\phi=0 \\
\text { on-shell }}}=\frac{1}{4} e_{i j}\left[\partial^{i} e_{k l} \partial^{j} e^{k l}-\partial^{i} e_{k l} \partial^{l} e^{k j}-\partial^{p} e^{i q} \partial^{j} e_{p q}\right. \\
\\
\left.\quad+\frac{1}{2} \alpha^{\prime}\left(\partial^{j q} e_{k l} \partial^{k l} e_{q}^{i}-\partial^{i p} e_{k l} \partial^{k l} e_{p}^{j}\right)+\frac{1}{12} \alpha^{\prime 2} \partial^{i j p q} e_{k l} \partial^{k l} e_{p q}\right],
\end{aligned}
$$

where we have made explicit the $\alpha^{\prime}$ factors in the various contributions. To compute the on-shell amplitude we pass to momentum space. We need not concern ourselves with overall normalization; all that matters here is the relative numerical factors between the two, four, and six-derivative terms. We thus have an on-shell amplitude $\mathcal{A}$ proportional to

$$
\begin{aligned}
\mathcal{A}=e_{1 i i^{\prime}} e_{2 j j^{\prime}} e_{3 k k^{\prime}}[- & k_{2}^{i} k_{3}^{i^{\prime}} \eta^{j k} \eta^{j^{\prime} k^{\prime}}+k_{2}^{i} k_{3}^{j^{\prime}} \eta^{j k} \eta^{i^{\prime} k^{\prime}}+k_{2}^{k} k_{3}^{i^{\prime}} \eta^{i j} \eta^{j^{\prime} k^{\prime}}+\text { permutations } \\
& +\frac{1}{2} \alpha^{\prime}\left(k_{2}^{i^{\prime}} k_{2}^{k^{\prime}} k_{3}^{j} k_{3}^{j^{\prime}} \eta^{i k}-k_{2}^{i} k_{2}^{k} k_{3}^{j} k_{3}^{j^{\prime}} \eta^{i^{\prime} k^{\prime}}\right)+\text { permutations } \\
& \left.-\frac{1}{12} \alpha^{\prime 2} k_{2}^{i} k_{2}^{i^{\prime}} k_{2}^{k} k_{2}^{k^{\prime}} k_{3}^{j} k_{3}^{j^{\prime}}+\text { permutations }\right]
\end{aligned}
$$

where we have used three different lines to list the terms with two, four, and six derivatives. By 'permutations' here we mean adding, in each line, the five copies with index permutations required to achieve full Bose symmetry. In order to show that the above has the conjectured factorized form we must rewrite the momentum factors in terms of momentum differences $k_{12}, k_{23}$, and $k_{31}$. This is possible because momentum factors must contract with polarization tensors, and using momentum conservation and transversality ensure they can be converted into momentum differences. For example,

$$
e_{2 j j^{\prime}} k_{1}^{j^{\prime}}=\frac{1}{2} e_{2 j j^{\prime}}\left(k_{1}^{j^{\prime}}+k_{1}^{j^{\prime}}\right)=\frac{1}{2} e_{2 j j^{\prime}}\left(k_{1}^{j^{\prime}}-k_{2}^{j^{\prime}}-k_{3}^{j^{\prime}}\right)=-\frac{1}{2} e_{2 j j^{\prime}} k_{31}^{j^{\prime}} .
$$

After rewriting all momenta as momentum differences the sum over permutations simplify and with modest work one can show that the two, four, and six derivative terms can be 
written as sum of products of the $T$ and $W$ tensors introduced in (2.3). Indeed, making use of (2.9) one finds,

$$
\begin{aligned}
\mathcal{A} & =\frac{1}{2} e_{1 i i^{\prime}}\left(k_{1}\right) e_{2 j j^{\prime}}\left(k_{2}\right) e_{3 k k^{\prime}}\left(k_{3}\right)\left[T^{i j k} T^{i^{\prime} j^{\prime} k^{\prime}}+\left(W^{i j k} T^{i^{\prime} j^{\prime} k^{\prime}}-T^{i j k} W^{i^{\prime} j^{\prime} k^{\prime}}\right)-W^{i j k} W^{i^{\prime} j^{\prime} k^{\prime}}\right] \\
& =\frac{1}{2} e_{1 i i^{\prime}}\left(k_{1}\right) e_{2 j j^{\prime}}\left(k_{2}\right) e_{3 k k^{\prime}}\left(k_{3}\right)\left(T^{i j k}+W^{i j k}\right)\left(T^{i^{\prime} j^{\prime} k^{\prime}}-W^{i^{\prime} j^{\prime} k^{\prime}}\right)
\end{aligned}
$$

in agreement with (2.5) and thus proving the claimed factorization.

\section{Conclusions and remarks}

Our work has determined the form (1.1) of the gauge invariant HSZ action that reproduces the on-shell cubic amplitudes of the theory. The $\mathcal{O}\left(\alpha^{\prime}\right)$ terms arise from the kinetic term for the three-form curvature $\widehat{H}$, which contains the Chern-Simons correction. Our work in section 4.3 reconfirmed that the cubic on-shell four-derivative couplings arise correctly see (4.34). The kinetic term $\widehat{H}^{2}$ also contains $\mathcal{O}\left(\alpha^{\prime 2}\right)$ contributions, but those would only affect six and higher-point amplitudes. The full HSZ action may contain other $\mathcal{O}\left(\alpha^{\prime}\right)$ terms that do not contribute to three-point amplitudes. The action includes the Riemann-cubed term derived in (4.39). Its coefficient is minus the coefficient of the same term in bosonic string theory. In bosonic string theory there is also a non-zero 'Gauss-Bonnet' Riemanncubed term, but its presence can only be seen from four-point amplitudes [20]. Neither the Riemann-cubed nor its related Gauss-Bonnet term are present in heterotic string theory. It would be interesting to see if the cubic-curvature Gauss-Bonnet interaction is present in HSZ theory. The physical effects of Riemann-cubed interactions were considered in [25] and, regardless of the sign of the term, they lead to causality violations that require the existence of new particles.

The action (1.1), while exactly gauge invariant, is unlikely to be exactly duality invariant. It is not, after all, the full action for HSZ theory. Reference [26] showed that the action (1.1), without the Riemann-cubed term, is not duality invariant to order $\alpha^{\prime}$ squared. It may be possible to use the methods in [26] to find out what other terms (that do not contribute to cubic amplitudes) are needed for duality invariance to order $\alpha^{\prime}$ squared. We continue to expect that, in terms of a metric and a $b$-field, an action with infinitely many terms is required for exact duality invariance.

We have not attempted to compute dilaton amplitudes from HSZ theory. There is no in-principle obstacle, and such computation could be done working in the Einstein frame. The graviton and dilaton fluctuations $\left(\hat{h}_{\mu \nu}, \hat{\phi}\right)$ with standard, decoupled, kinetic terms are linear combinations of the fluctuations $\left(h_{\mu \nu}, \phi\right)$ that we use. These redefinitions must be performed to compute physical dilaton amplitudes. They were not needed to compute gravity and $b$-field amplitudes because $h_{\mu \nu}$ differs from $\hat{h}_{\mu \nu}$ only by dilaton dependent terms and the dilatons differ from each other by traces of $h$, which do not contribute for on-shell three-point amplitudes.

The computation of quartic amplitudes in HSZ theory is clearly a very interesting challenge. World-sheet methods may give an efficient way to obtain answers. It is still important, however, to develop techniques to compute amplitudes in a theory with a double 
metric. The HSZ action is not uniquely fixed by the gauge structure of the theory [10]: one can add higher-order gauge-invariant products of the tensor field $\mathcal{T}$ which are expected to modify quartic and higher-order amplitudes. In those theories, the spacetime action would be the natural tool to compute amplitudes, and one could wonder how the conformal field theory method would work. In this paper we have taken the first steps in the computation of amplitudes starting from a theory with a double metric. The computation of four-point amplitudes and of amplitudes that involve dilatons would be significant progress.

It is natural to ask to what degree global duality determines the classical effective action for the massless fields of string theory. Additionally, given an effective field theory of metric, $b$-field and dilaton, it is also natural to ask if the theory has a duality symmetry. HSZ theory is useful as it is the simplest gravitational theory with higher derivative corrections and exact global duality. By investigating HSZ theory we will better understand the constraints of duality and its role in the effective field theory of strings.

\section{Acknowledgments}

We thank Olaf Hohm for collaboration in early stages of this work and for useful comments. This work is supported by the U.S. Department of Energy under grant Contract Number DE-SC0012567.

Open Access. This article is distributed under the terms of the Creative Commons Attribution License (CC-BY 4.0), which permits any use, distribution and reproduction in any medium, provided the original author(s) and source are credited.

\section{References}

[1] G. Veneziano, Scale factor duality for classical and quantum strings, Phys. Lett. B 265 (1991) 287 [INSPIRE].

[2] K.A. Meissner and G. Veneziano, Symmetries of cosmological superstring vacua, Phys. Lett. B 267 (1991) 33 [INSPIRE].

[3] A. Sen, $O(d) \otimes O(d)$ symmetry of the space of cosmological solutions in string theory, scale factor duality and two-dimensional black holes, Phys. Lett. B 271 (1991) 295 [INSPIRE].

[4] S.F. Hassan and A. Sen, Twisting classical solutions in heterotic string theory, Nucl. Phys. B 375 (1992) 103 [hep-th/9109038] [INSPIRE].

[5] W. Siegel, Superspace duality in low-energy superstrings, Phys. Rev. D 48 (1993) 2826 [hep-th/9305073] [INSPIRE].

[6] W. Siegel, Manifest duality in low-energy superstrings, hep-th/9308133 [INSPIRE].

[7] C. Hull and B. Zwiebach, Double Field Theory, JHEP 09 (2009) 099 [arXiv:0904.4664] [INSPIRE].

[8] O. Hohm, C. Hull and B. Zwiebach, Background independent action for double field theory, JHEP 07 (2010) 016 [arXiv: 1003.5027] [INSPIRE].

[9] O. Hohm, C. Hull and B. Zwiebach, Generalized metric formulation of double field theory, JHEP 08 (2010) 008 [arXiv: 1006.4823] [INSPIRE]. 
[10] O. Hohm, W. Siegel and B. Zwiebach, Doubled $\alpha^{\prime}$-geometry, JHEP 02 (2014) 065 [arXiv: 1306.2970] [INSPIRE].

[11] O. Hohm and B. Zwiebach, Double Metric, Generalized Metric and $\alpha^{\prime}$-Geometry, arXiv: 1509.02930 [INSPIRE].

[12] O. Hohm and B. Zwiebach, Green-Schwarz mechanism and $\alpha^{\prime}$-deformed Courant brackets, JHEP 01 (2015) 012 [arXiv: 1407.0708] [INSPIRE].

[13] D. Marques and C.A. Núñez, T-duality and $\alpha^{\prime}$-corrections, JHEP 10 (2015) 084 [arXiv: 1507.00652] [INSPIRE].

[14] W. Siegel, Amplitudes for left-handed strings, arXiv:1512.02569 [INSPIRE].

[15] O. Hohm, On factorizations in perturbative quantum gravity, JHEP 04 (2011) 103 [arXiv: 1103.0032] [INSPIRE].

[16] R.H. Boels and C. Horst, Perturbative quantum gravity in double field theory, arXiv: 1512.03192 [INSPIRE].

[17] D.J. Gross, J.A. Harvey, E.J. Martinec and R. Rohm, Heterotic String Theory. 2. The Interacting Heterotic String, Nucl. Phys. B 267 (1986) 75 [INSPIRE].

[18] B. Zwiebach, Curvature Squared Terms and String Theories, Phys. Lett. B 156 (1985) 315 [INSPIRE].

[19] R.R. Metsaev and A.A. Tseytlin, Order alpha-prime (Two Loop) Equivalence of the String Equations of Motion and the $\sigma$-model Weyl Invariance Conditions: Dependence on the Dilaton and the Antisymmetric Tensor, Nucl. Phys. B 293 (1987) 385 [InSPIRE].

[20] R.R. Metsaev and A.A. Tseytlin, Curvature Cubed Terms in String Theory Effective Actions, Phys. Lett. B 185 (1987) 52 [InSPIRE].

[21] O. Hohm and B. Zwiebach, Double field theory at order $\alpha^{\prime}$, JHEP 11 (2014) 075 [arXiv: 1407.3803] [INSPIRE].

[22] J. Scherk and J.H. Schwarz, Dual Models for Nonhadrons, Nucl. Phys. B 81 (1974) 118 [INSPIRE].

[23] J. Polchinski, String theory. Vol. 1: An introduction to the bosonic string, Cambridge University Press (1998).

[24] J. Polchinski, String theory. Vol. 2: Superstring theory and beyond, Cambridge University Press (1998).

[25] X.O. Camanho, J.D. Edelstein, J. Maldacena and A. Zhiboedov, Causality Constraints on Corrections to the Graviton Three-Point Coupling, JHEP 02 (2016) 020 [arXiv:1407.5597] [INSPIRE].

[26] O. Hohm and B. Zwiebach, T-duality Constraints on Higher Derivatives Revisited, arXiv: 1510.00005 [INSPIRE]. 